\title{
The Reciprocal Sums of the Pell Numbers
}

\author{
Fuling Zhang* \\ School of Mathematics and Physics, Weinan Normal University, Weinan, Shaanxi, 714099, P.R.China \\ *Corresponding author
}

\begin{abstract}
In this paper, we consider the reciprocal sums of the Pell numbers by elementary methods. First, we investigate the reciprocals sums of two products of Pell numbers. Then, we study the alternating reciprocals sums of two products of Pell numbers .Last, we consider the reciprocal sums of even and odd terms in the Pell sequence. We obtain some interesting identities involving the Pell numbers.
\end{abstract}

\section{Keywords—pell numbers; reciprocal sum; identity}

\section{INTRODUCTION}

Fnis defined by the second-order linear recurrence sequences

$$
\mathrm{Fn}+2=\mathrm{Fn}+1+\mathrm{Fn}, \mathrm{F} 0=0, \mathrm{~F} 1=1,(1.1)
$$

The Fibonacci numbers can be defined by Binet's formulae $\mathrm{Fn}=\alpha \mathrm{n}-\beta \mathrm{n} \alpha-\beta$, where $\alpha=1+52, \beta=1-52$,

There are many interesting results on the properties of Fibonacci sequences; see [1-6]. Ohtsuka and Nakamura [1] studied the properties of the Fibonacci numbers and proved the following two interesting identities:

$$
\begin{gathered}
\mathrm{k}=\mathrm{n} \infty 1 \mathrm{Fk}-1=\mathrm{Fn}-2, \quad \text { if } \mathrm{n} \text { is even and } \mathrm{n} \geq 2, \mathrm{Fn}-2-1 \text {, if } \\
\mathrm{n} \text { is odd and } \mathrm{n} \geq 1 \text {. }
\end{gathered}
$$

$\mathrm{k}=\mathrm{n} \infty 1 \mathrm{Fk} 2-1=\mathrm{FnFn}-1-1, \quad$ if $\mathrm{n}$ is even and $\mathrm{n} \geq 2, \mathrm{FnFn}-1$, if $\mathrm{n}$ is odd and $\mathrm{n} \geq 1$.

$\mathrm{x}$ is the floor function; that is, it denotes the greatest integer less than or equal to $\mathrm{x}$.

Wang AYZ, Zhang F. [3] researched the properties of the Fibonacci numbers and proved the following four interesting identities:

$$
\mathrm{k}=\mathrm{nmn} 1 \mathrm{~F} 2 \mathrm{k}-1=\mathrm{F} 2 \mathrm{n}-1, \quad \text { if } \mathrm{m}=2 \text { and }
$$

$\mathrm{n} \geq 3, \mathrm{~F} 2 \mathrm{n}-1-1$, if $\mathrm{m} \geq 3$ and $\mathrm{n} \geq 1$.

$\mathrm{k}=\mathrm{nmn} 1 \mathrm{~F} 2 \mathrm{k}-1-1=\mathrm{F} 2 \mathrm{n}-2$, For all $\mathrm{n} \geq 1$ and $\mathrm{m} \geq 2$.

$\mathrm{k}=\mathrm{nmn} 1 \mathrm{~F} 2 \mathrm{k} 2-1=\mathrm{F} 4 \mathrm{n}-2$, For all $\mathrm{n} \geq 1$ and $\mathrm{m} \geq 2$.

$\mathrm{k}=\mathrm{nmn} 1 \mathrm{~F} 2 \mathrm{k}-12-1=\mathrm{F} 4 \mathrm{n}-4$, For all $\mathrm{n} \geq 1$ and $\mathrm{m} \geq 2$.

Wang AYZ, Liu RN [4] demonstrated the following identities for the Fibonacci numbers

$$
\begin{gathered}
\mathrm{k}=\mathrm{nmn} 1 \mathrm{FkFk}-1-1=\mathrm{Fn} 2, \\
\text { if } \mathrm{n} \text { is odd. }
\end{gathered}
$$

$\mathrm{k}=\mathrm{nmn} 1 \mathrm{~F} 2 \mathrm{kF} 2 \mathrm{k}-1-1=\mathrm{F} 4 \mathrm{n}-3$, For all $\mathrm{n} \geq 2$ and $\mathrm{m} \geq 2$.

$\mathrm{Pn}$ is also defined by the second-order are

$\mathrm{Pn}+2=2 \mathrm{Pn}+1+\mathrm{Pn}, \mathrm{P} 0=0, \mathrm{P} 1=1$.

The Pell numbers also provide boundless opportunities to experiment, explore, and conjecture, they are a lot of fun for inquisitive amateurs and professionals alike. The authors [7] and [8] studied the infinite sums derived from the Pell numbers and proved the following identities:

$\mathrm{k}=\mathrm{n} \infty 1 \mathrm{Pk}-1=\mathrm{Pn}-1+\mathrm{Pn}-2, \quad$ if $\mathrm{n}$ is even and $\mathrm{n} \geq 2, \mathrm{Pn}-1+\mathrm{Pn}-2-1 \quad$, if $\mathrm{n}$ is odd and $\mathrm{n} \geq 1$.

$\mathrm{k}=\mathrm{n} \infty 1 \mathrm{Pk} 2-1=2 \mathrm{Pn}-1+\mathrm{Pn}-1, \quad$ if $\mathrm{n}$ is even and $\mathrm{n} \geq 2,2 \mathrm{Pn}-1+\mathrm{Pn} \quad, \quad$ if $\mathrm{n}$ is odd and $\mathrm{n} \geq 1$. (1.13)

$\mathrm{Xu}$ and Wang [9] proofed the following interesting identities for the Pell numbers:

$$
\begin{aligned}
\mathrm{k}=\mathrm{n} \infty 1 \mathrm{Pk} 3-1=\mathrm{Pn} 2 \mathrm{Pn}-1+3 \mathrm{PnPn}-12+-6182 \mathrm{Pn}-9182 \mathrm{Pn}-1 \text {, } \\
\text { if } \mathrm{n} \text { is even and }
\end{aligned}
$$

$\mathrm{n}>2$, $\mathrm{Pn} 2 \mathrm{Pn}-1+3 \mathrm{PnPn}-12+-6182 \mathrm{Pn}-9182 \mathrm{Pn}-1$, if $\mathrm{n}$ is odd and $n \geq 1$.

Applying elementary methods, we investigate the partial finite sums of the Pell numbers in this paper, and obtain some interesting families of identities. In section 2, we consider the sums of products of two reciprocals. In section 3, we study the alternating sums of products of two reciprocals. In section 4, we consider the reciprocal sums of even and odd terms in the Pell sequence.

\section{RESULTS I: THE RECIPROCAL SUMS}

$\mathrm{x} 2-2 \mathrm{x}-1=0$, the Pell numbers can be defined by Binet-like formulae:

$$
\mathrm{Pn}=\gamma \mathrm{n}-\delta \mathrm{n} \gamma-\delta, \text { where } \gamma=1+2, \delta=1-2
$$

Using the Binet-like formulae, we can obtain properties of Pell numbers:

Lemma 2.1 Let $m, n, s, t$ be positive integers

$$
P_{m} P_{n}-P_{s} P_{t}=(-1)^{m+1} P_{n-s} P_{n-t},(n>\max \{s, t\}, m+n=s+t)
$$

Lemma 2.2

$$
P_{m} P_{n}+P_{m+1} P_{n+1}=P_{m+n+1} .
$$


As consequence of (2.2), we have the following result:

Corollary 2.3

$P_{n}^{2}+P_{n+1}^{2}=P_{2 n+1}$.

Corollary 2.4

$P_{n-1} P_{n+1}+P_{n} P_{n+2}=P_{2 n+1}$

Lemma 2.5

$$
P_{n+1} P_{n+2}-P_{n-1} P_{n}=2 P_{2 n+1}
$$

Proof Applying (2.4) and recursion formula of Pell numbers

$$
\begin{aligned}
2 P_{2 n+1} & =2 P_{n-1} P_{n+1}+2 P_{n} P_{n+2} \\
& =\left(P_{n+2}-P_{n}\right) P_{n-1}+\left(P_{n+1}-P_{n-1}\right) P_{n+2} \\
& =P_{n+1} P_{n+2}-P_{n} P_{n-1}
\end{aligned}
$$

Lemma 2.6

$$
P_{n+2}^{2}-P_{n}^{2}=2 P_{2 n+2}
$$

Proof Applying (2.3)

$$
\begin{aligned}
P_{n+2}^{2}-P_{n}^{2} & =P_{2 n+3}-P_{n+1}^{2}-P_{n}^{2} \\
& =P_{2 n+3}-\left(P_{n+1}^{2}+P_{n}^{2}\right) \\
& =P_{2 n+3}-P_{2 n+1} \\
& =2 P_{2 n+2}
\end{aligned}
$$

A. Reciprocal Sum of $P_{k} P_{k+1}$

$$
\begin{gathered}
\text { Lemma } 2.7 \text { If } n \geq 1, \\
2 P_{2 n+1}^{2}+1>2\left(P_{n+1}^{2}+1\right)^{2}>P_{n} P_{n+1}\left(2 P_{n+1}^{2}+1\right)
\end{gathered}
$$

Proof applying (2.3)

$2 P_{2 n+1}^{2}+1=2\left(P_{n}^{2}+P_{n+1}^{2}\right)^{2}+1>2 P_{n+1}^{4}+4 P_{n+1}^{2}+2=2\left(P_{n+1}^{2}+1\right)^{2}$,

Therefore

$2 P_{2 n+1}^{2}+1>2\left(P_{n+1}^{2}+1\right)^{2}>2\left(P_{n+1}^{2}+1\right) P_{n} P_{n+1}>P_{n} P_{n+1}\left(2 P_{n+1}^{2}+1\right)$.
Then

$$
\left\lfloor\left(\sum_{k=n}^{m n} \frac{1}{P_{k} P_{k+1}}\right)^{-1}\right\rfloor=\left\{\begin{array}{c}
2 P_{n}^{2}, \text { if } n \text { is even } \\
2 P_{n}^{2}-1, \text { if } n \text { is odd }
\end{array}\right.
$$

Proof applying (2.1)

$$
\frac{1}{2 P_{k}^{2}}-\frac{1}{P_{k} P_{k+1}}-\frac{1}{2 P_{k+1}^{2}}=\frac{P_{k+1}^{2}-2 P_{k} P_{k+1}-P_{k}^{2}}{2 P_{k}^{2} P_{k+1}^{2}}=\frac{P_{k-1} P_{k+1}-P_{k}^{2}}{2 P_{k}^{2} P_{k+1}^{2}}=\frac{(-1)^{k}}{2 P_{k}^{2} P_{k+1}^{2}},
$$

Therefore

$$
\frac{1}{P_{k} P_{k+1}}=\frac{1}{2 P_{k}^{2}}-\frac{1}{2 P_{k+1}^{2}}+\frac{(-1)^{k-1}}{2 P_{k}^{2} P_{k+1}^{2}}
$$

Now we have

$$
\sum_{k=n}^{m n} \frac{1}{P_{k} P_{k+1}}=\frac{1}{2 P_{n}^{2}}-\frac{1}{2 P_{m n+1}^{2}}+\sum_{k=n}^{m n} \frac{(-1)^{k-1}}{2 P_{k}^{2} P_{k+1}^{2}},
$$

If $\mathrm{n}$ is even, it is easy to see that

$$
\sum_{k=n}^{m n} \frac{(-1)^{k-1}}{2 P_{k}^{2} P_{k+1}^{2}}<0
$$

By the above equation (2.10)

$$
\sum_{k=n}^{m n} \frac{1}{P_{k} P_{k+1}}<\frac{1}{2 P_{n}^{2}}
$$

For any $k \geq 1$

$$
\begin{aligned}
\frac{1}{2 P_{k}^{2}+1}-\frac{1}{P_{k} P_{k+1}}-\frac{1}{2 P_{k+1}^{2}+1} & =\frac{\left(2 P_{k+1}^{2}+1\right) P_{k} P_{k+1}-\left(2 P_{k}^{2}+1\right)\left(2 P_{k+1}^{2}+1\right)-\left(2 P_{k}^{2}+1\right) P_{k} P_{k+1}}{\left(2 P_{k}^{2}+1\right) P_{k} P_{k+1}\left(2 P_{k+1}^{2}+1\right)} \\
& =\frac{\left(2 P_{k+1}^{2}-2 P_{k}^{2}\right) P_{k} P_{k+1}-\left(2 P_{k}^{2}+1\right)\left(2 P_{k+1}^{2}+1\right)}{\left(2 P_{k}^{2}+1\right) P_{k} P_{k+1}\left(2 P_{k+1}^{2}+1\right)} \\
& =\frac{(-1)^{k} 2 P_{k} P_{k+1}-2 P_{k}^{2}-2 P_{k+1}^{2}-1}{\left(2 P_{k}^{2}+1\right) P_{k} P_{k+1}\left(2 P_{k+1}^{2}+1\right)}<0 .
\end{aligned}
$$

Then $\frac{1}{P_{k} P_{k+1}}=\frac{1}{2 P_{k}^{2}+1}-\frac{1}{2 P_{k+1}^{2}+1}+\frac{(-1)^{k-1} 2 P_{k} P_{k+1}+2 P_{k}^{2}+2 P_{k+1}^{2}+1}{\left(2 P_{k}^{2}+1\right) P_{k} P_{k+1}\left(2 P_{k+1}^{2}+1\right)}$,

So 


$$
\begin{aligned}
\sum_{k=n}^{m n} \frac{1}{P_{k} P_{k+1}} & =\frac{1}{2 P_{n}^{2}+1}-\frac{1}{2 P_{m n+1}^{2}+1}+\sum_{k=n}^{m n} \frac{(-1)^{k-1} 2 P_{k} P_{k+1}+2 P_{k}^{2}+2 P_{k}^{2}+1}{\left(2 P_{k}^{2}+1\right) P_{k} P_{k+1}\left(2 P_{k+1}^{2}+1\right)} \\
& >\frac{1}{2 P_{n}^{2}+1}-\frac{1}{2 P_{2 n+1}^{2}+1}+\frac{(-1)^{n-1} 2 P_{n} P_{n+1}+2 P_{n}^{2}+2 P_{n+1}^{2}+1}{\left(2 P_{n}^{2}+1\right) P_{n} P_{n+1}\left(2 P_{n+1}^{2}+1\right)} \\
& >\frac{1}{2 P_{n}^{2}+1}-\frac{1}{2 P_{2 n+1}^{2}+1}+\frac{1}{P_{n} P_{n+1}\left(2 P_{n+1}^{2}+1\right)},
\end{aligned}
$$

Using (2.7)

$$
\sum_{k=n}^{m n} \frac{1}{P_{k} P_{k+1}}>\frac{1}{2 P_{n}^{2}+1}
$$

Combining (2.11) and (2.13), we have

$$
\frac{1}{2 P_{n}^{2}+1}<\sum_{k=n}^{m n} \frac{1}{P_{k} P_{k+1}}<\frac{1}{2 P_{n}^{2}}
$$

which means that the statement is true when $\mathrm{n}$ is even

$$
\left\lfloor\left(\sum_{k=n}^{m n} \frac{1}{P_{k} P_{k+1}}\right)^{-1}\right\rfloor=2 P_{n}^{2} .
$$

If $\mathrm{n}$ is odd, a similar calculation show that, for $k \geq 1$

$$
\begin{aligned}
\frac{1}{2 P_{k}^{2}-1}-\frac{1}{P_{k} P_{k+1}}-\frac{1}{2 P_{k+1}^{2}-1} & =\frac{\left(2 P_{k+1}^{2}-1\right) P_{k} P_{k+1}-\left(2 P_{k}^{2}-1\right)\left(2 P_{k+1}^{2}-1\right)-\left(2 P_{k}^{2}-1\right) P_{k} P_{k+1}}{\left(2 P_{k}^{2}-1\right) P_{k} P_{k+1}\left(2 P_{k+1}^{2}-1\right)} \\
& =\frac{\left(2 P_{k+1}^{2}-2 P_{k}^{2}\right) P_{k} P_{k+1}-\left(2 P_{k}^{2}-1\right)\left(2 P_{k+1}^{2}-1\right)}{\left(2 P_{k}^{2}-1\right) P_{k} P_{k+1}\left(2 P_{k+1}^{2}-1\right)} \\
& =\frac{(-1)^{k} 2 P_{k} P_{k+1}+2 P_{k}^{2}+2 P_{k+1}^{2}-1}{\left(2 P_{k}^{2}-1\right) P_{k} P_{k+1}\left(2 P_{k+1}^{2}-1\right)}>0,
\end{aligned}
$$

So

$$
\frac{1}{P_{k} P_{k+1}}=\frac{1}{2 P_{k}^{2}-1}-\frac{1}{2 P_{k+1}^{2}-1}-\frac{(-1)^{k} 2 P_{k} P_{k+1}+2 P_{k}^{2}+2 P_{k+1}^{2}-1}{\left(2 P_{k}^{2}-1\right) P_{k} P_{k+1}\left(2 P_{k+1}^{2}-1\right)},
$$

From which we get

$\sum_{k=n}^{m n} \frac{1}{P_{k} P_{k+1}}=\frac{1}{2 P_{n}^{2}-1}-\frac{1}{2 P_{m n+1}^{2}-1}-\sum_{k=n}^{m n} \frac{(-1)^{k} 2 P_{k} P_{k+1}+2 P_{k}^{2}+2 P_{k+1}^{2}-1}{\left(2 P_{k}^{2}-1\right) P_{k} P_{k+1}\left(2 P_{k+1}^{2}-1\right)}$,

Hence

$$
\sum_{k=n}^{m n} \frac{1}{P_{k} P_{k+1}}<\frac{1}{2 P_{n}^{2}-1}
$$

With (2.10) and (2.6),

$$
\begin{aligned}
\sum_{k=n}^{m} \frac{1}{P_{k} P_{k+1}} & =\frac{1}{2 P_{n}^{2}}-\frac{1}{2 P_{m+1}^{2}}+\sum_{k=n}^{m} \frac{(-1)^{k-1}}{2 P_{k}^{2} P_{k+1}^{2}} \\
& >\frac{1}{2 P_{n}^{2}}-\frac{1}{2 P_{2 n+1}^{2}}+\frac{1}{2 P_{n}^{2} P_{n+1}^{2}}-\frac{1}{2 P_{n+1}^{2} P_{n+2}^{2}}=\frac{1}{2 P_{n}^{2}}+\frac{2 P_{2 n+2}}{2 P_{n}^{2} P_{n+1}^{2} P_{n+2}^{2}}-\frac{2 P_{2 n+2}}{2^{2} P_{2 n+1}^{2} P_{2 n+2}} .
\end{aligned}
$$

According to (2.2) and (2.3)

$$
P_{2 n+1}=P_{n-1} P_{n+1}+P_{n} P_{n+2}=P_{n}^{2}+P_{n+1}^{2},
$$

So

$$
P_{2 n+1}>P_{n} P_{n+2, \text { and }} P_{2 n+2}>P_{2 n+1}>P_{n+1}^{2},
$$

Hence

$$
2^{2} P_{2 n+2} P_{2 n+1}^{2}>2 P_{n}^{2} P_{n+1}^{2} P_{n+2}^{2},
$$

As a result

$$
\sum_{k=n}^{m n} \frac{1}{P_{k} P_{k+1}}>\frac{1}{2 P_{n}^{2}}
$$

Combining (2.11) and (2.13),

$$
\frac{1}{2 P_{n}^{2}}<\sum_{k=n}^{m n} \frac{1}{P_{k} P_{k+1}}<\frac{1}{2 P_{n}^{2}-1}
$$

When $\mathrm{n}$ is odd, that the statement is true

$$
\left\lfloor\left(\sum_{k=n}^{m n} \frac{1}{P_{k} P_{k+1}}\right)^{-1}\right\rfloor=2 P_{n}^{2}-1 .
$$

On the basis of (2.14) and (2.18), we have the theorem 2.8.

\section{Corollary 2.9}

$$
\left\lfloor\left(\sum_{k=n}^{\infty} \frac{1}{P_{k} P_{k+1}}\right)^{-1}\right\rfloor=\left\{\begin{array}{c}
2 P_{n}^{2} \text {, if } n \text { is even } \\
2 P_{n}^{2}-1, \text { if } n \text { is odd }
\end{array}\right.
$$

Proof applying (2.8), if $\mathrm{n}$ is even

$$
\frac{1}{2 P_{k}^{2}}-\frac{1}{P_{k} P_{k+1}}-\frac{1}{2 P_{k+1}^{2}}=\frac{(-1)^{k}}{2 P_{k}^{2} P_{k+1}^{2}}>0
$$

Hence 
$\frac{1}{2 P_{k}^{2}}>\frac{1}{P_{k} P_{k+1}}+\frac{1}{2 P_{k+1}^{2}}>\frac{1}{P_{k} P_{k+1}}+\frac{1}{P_{k+1} P_{k+2}}+\frac{1}{2 P_{k+2}^{2}}>\ldots \ldots$

So

$$
\sum_{k=n}^{\infty} \frac{1}{P_{k} P_{k+1}}<\frac{1}{2 P_{n}^{2}}
$$

In line with (2.12)

$$
\frac{1}{2 P_{k}^{2}+1}-\frac{1}{P_{k} P_{k+1}}-\frac{1}{2 P_{k+1}^{2}+1}<0
$$

Therefore

$\frac{1}{2 P_{k}^{2}+1}<\frac{1}{P_{k} P_{k+1}}+\frac{1}{2 P_{k+1}^{2}+1}<\frac{1}{P_{k} P_{k+1}}+\frac{1}{P_{k+1} P_{k+2}}+\frac{1}{2 P_{k+2}^{2}+1}<\ldots \ldots$

As a result

$$
\frac{1}{2 P_{n}^{2}+1}<\sum_{k=n}^{\infty} \frac{1}{P_{k} P_{k+1}}
$$

Combining (2.19) and (2.20), when $\mathrm{n}$ is even, the statement is true

$$
\frac{1}{2 P_{n}^{2}+1}<\sum_{k=n}^{\infty} \frac{1}{P_{k} P_{k+1}}<\frac{1}{2 P_{n}^{2}}
$$

With (2.8), when $\mathrm{n}$ is odd,

$$
\frac{1}{2 P_{k}^{2}}-\frac{1}{P_{k} P_{k+1}}-\frac{1}{2 P_{k+1}^{2}}=\frac{(-1)^{k}}{2 P_{k}^{2} P_{k+1}^{2}}<0
$$

So

$\frac{1}{2 P_{k}^{2}}<\frac{1}{P_{k} P_{k+1}}+\frac{1}{2 P_{k+1}^{2}}<\frac{1}{P_{k} P_{k+1}}+\frac{1}{P_{k+1} P_{k+2}}+\frac{1}{2 P_{k+2}^{2}}<\ldots \ldots$

Hence

$$
\frac{1}{2 P_{k}^{2}-1}-\frac{1}{P_{k} P_{k+1}}-\frac{1}{2 P_{k+1}^{2}-1}>0
$$

Therefore

$$
\frac{1}{2 P_{k}^{2}-1}>\frac{1}{G_{k} G_{k+1}}+\frac{1}{2 P_{k+1}^{2}-1}>\frac{1}{P_{k} P_{k+1}}+\frac{1}{P_{k+1} P_{k+2}}+\frac{1}{2 P_{k+2}^{2}-1}>\ldots \ldots
$$

So

$$
\sum_{k=n}^{\infty} \frac{1}{P_{k} P_{k+1}}<\frac{1}{2 P_{n}^{2}-1}
$$

Combining (2.22) and (2.23), when $\mathrm{n}$ is odd, the statement is true

$$
\frac{1}{2 P_{n}^{2}}<\sum_{k=n}^{\infty} \frac{1}{P_{k} P_{k+1}}<\frac{1}{2 P_{n}^{2}-1}
$$
2.9 .

Accordance with (2.21) and (2.24), we have the Corollary

III. MAin Results II: Alternating ReCiPROCAL SuMS

Lemma 3.1

$$
\frac{P_{2 n+2}}{P_{2 n+1}}-\frac{P_{m n+2}}{P_{m n+1}}<0
$$

Proof applying (2.1),

$$
\frac{P_{2 n+2}}{P_{2 n+1}}-\frac{P_{m n+2}}{P_{m n+1}}=\frac{P_{2 n+2} P_{m n+1}-P_{2 n+1} P_{m n+2}}{P_{2 n+1} P_{m n+1}}=(-1)^{2 n+1} \frac{P_{(m-2) n+1}}{P_{2 n+1} P_{m n+1}}<0 .
$$

As a similar way, we have

\section{Lemma 3.2}

$$
\frac{P_{2 n+1}}{P_{2 n}}-\frac{P_{m n+2}}{P_{m n+1}}<0
$$

Lemma 3.3

$$
\frac{1}{2 P_{n}^{2}}<\sum_{k=n}^{\infty} \frac{1}{P_{k} P_{k+1}}
$$

$$
\frac{1}{P_{2 n}-1}-\frac{P_{n+1}}{P_{n} P_{2 n+1}}=\frac{(-1)^{n+1} P_{n}+P_{n+1}}{\left(P_{2 n}-1\right) P_{n} P_{2 n+1}}>0
$$

On the basis of (2.15) 
Lemma 3.4

$$
\frac{1}{P_{2 n}+1}-\frac{P_{n+1}}{P_{n} P_{2 n+1}}=\frac{(-1)^{n+1} P_{n}-P_{n+1}}{\left(P_{2 n}-1\right) P_{n} P_{2 n+1}}<0
$$

\section{Lemma 3.5}

$$
\frac{P_{n+1}}{P_{n}}-\frac{1}{P_{2 n}}=\frac{P_{2 n+1}+(-1)^{n+1}-1}{P_{2 n}}
$$

Proof combining (2.1), (2.2) and (2.3),

$$
\begin{aligned}
\frac{P_{n+1}}{P_{n}}-\frac{1}{P_{2 n}} & =\frac{P_{2 n} P_{n+1}-P_{n}}{P_{n} P_{2 n}}=\frac{\left(P_{n-1} P_{n}+P_{n} P_{n+1}\right) P_{n+1}-P_{n}}{P_{n} P_{2 n}}=\frac{P_{n-1} P_{n+1}+P_{n+1}^{2}-1}{P_{2 n}} \\
& =\frac{\left(P_{n-1} P_{n+1}-P_{n}^{2}\right)+\left(P_{n}^{2}+P_{n+1}^{2}\right)-1}{P_{2 n}}=\frac{P_{2 n+1}+(-1)^{n}-1}{P_{2 n}} .
\end{aligned}
$$

Use the same way, we have

\section{Lemma 3.6}

$$
\frac{P_{n+1}}{P_{n}}+\frac{1}{P_{2 n}}=\frac{P_{2 n+1}+(-1)^{n}+1}{P_{2 n}} .
$$

\section{Theorem}

$$
\left\lfloor\left(\sum_{k=n}^{m n} \frac{(-1)^{k}}{P_{k} P_{k+1}}\right)^{-1}\right\rfloor=\left\{\begin{array}{l}
P_{2 n}-1, \text { if } n \text { is even } \\
-P_{2 n}-1, \text { if } n \text { is odd }
\end{array} .\right.
$$

Proof applying (2.1),

$$
\frac{(-1)^{k}}{P_{k} P_{k+1}}=\frac{P_{k+1}^{2}-P_{k} P_{k+2}}{P_{k} P_{k+1}}=\frac{P_{k+1}}{P_{k}}-\frac{P_{k+2}}{P_{k+1}},
$$

So

$$
\sum_{k=n}^{m n} \frac{(-1)^{k}}{P_{k} P_{k+1}}=\sum_{k=n}^{m n}\left(\frac{P_{k+1}}{P_{k}}-\frac{P_{k+2}}{P_{k+1}}\right)=\frac{P_{n+1}}{P_{n}}-\frac{P_{m n+2}}{P_{m n+1}}
$$

If $\mathrm{n}$ is even, with (3.1) and (2.1)

$\frac{P_{n+1}}{P_{n}}-\frac{P_{m n+2}}{P_{m n+1}}<\frac{P_{n+1}}{P_{n}}-\frac{P_{2 n+2}}{P_{2 n+1}}=\frac{P_{2 n+1} P_{n+1}-P_{2 n+2} P_{n}}{P_{n} P_{2 n+1}}=\frac{(-1)^{n} P_{n+1}}{P_{n} P_{2 n+1}}=\frac{P_{n+1}}{P_{n} P_{2 n+1}}$,

On the basis of (3.3)

$$
\frac{P_{n+1}}{P_{n}}-\frac{P_{m n+2}}{P_{m n+1}}<\frac{P_{n+1}}{P_{n} P_{2 n+1}}<\frac{1}{P_{2 n}-1}
$$

According to (3.5) and (3.2)

$$
\begin{gathered}
\frac{P_{n+1}-\frac{1}{P_{n}}-\frac{P_{m n+2}}{P_{2 n}}=}{P_{m n+1}} \frac{P_{2 n+1}}{P_{2 n}}-\frac{P_{m n+2}}{P_{m n+1}}+\frac{(-1)^{n+1}-1}{P_{2 n}}=\frac{P_{2 n+1}}{P_{2 n}}-\frac{P_{m n+2}}{P_{m n+1}} ; \\
\frac{P_{n+1}}{P_{n}}-\frac{P_{m n+2}}{P_{m n+1}}>\frac{1}{P_{2 n}},
\end{gathered}
$$

Combining(3.7), (3.8)and(3.9), when $\mathrm{n}$ is even, the statement is true

$$
\left\lfloor\left(\sum_{k=n}^{m n} \frac{(-1)^{k}}{P_{k} P_{k+1}}\right)^{-1}\right\rfloor=P_{2 n}-1 .
$$

If $\mathrm{n}$ is even, with (3.1) and (2.1)

$$
\frac{P_{n+1}}{P_{n}}-\frac{P_{m n+2}}{P_{m n+1}}<\frac{P_{n+1}}{P_{n}}-\frac{P_{2 n+2}}{P_{2 n+1}}=\frac{P_{2 n+1} P_{n+1}-P_{2 n+2} P_{n}}{P_{n} P_{2 n+1}}=\frac{(-1)^{n} P_{n+1}}{P_{n} P_{2 n+1}}=-\frac{P_{n+1}}{P_{n} P_{2 n+1}},
$$

Applying (3.4)

$$
\frac{P_{n+1}}{P_{n}}-\frac{P_{m n+2}}{P_{m n+1}}<-\frac{1}{P_{2 n}+1},
$$

Using (3.6) and (3.2)

$$
\begin{aligned}
& \frac{P_{n+1}}{P_{n}}+\frac{1}{P_{2 n}}=\frac{P_{2 n+1}+(-1)^{n+1}+1}{P_{2 n}}=\frac{P_{2 n+1}}{P_{2 n}}, \\
& \frac{P_{n+1}}{P_{n}}+\frac{1}{P_{2 n}}-\frac{P_{m n+2}}{P_{m n+1}}=\frac{P_{2 n+1}}{P_{2 n}}-\frac{P_{m n+2}}{P_{m n+1}}>0,
\end{aligned}
$$

Hence

$$
\frac{P_{n+1}}{P_{n}}-\frac{P_{m n+2}}{P_{m n+1}}>-\frac{1}{P_{2 n}}
$$

Combining (3.7), (3.11) and (3.12), when $\mathrm{n}$ is odd, the statement is true

$$
-\frac{1}{P_{2 n}}<\sum_{k=n}^{m n} \frac{(-1)^{k}}{P_{k} P_{k+1}}=\frac{P_{n+1}}{P_{n}}-\frac{P_{m n+2}}{P_{m n+1}}<-\frac{1}{P_{2 n}+1},
$$


Therefore

$$
\left\lfloor\left(\sum_{k=n}^{m n} \frac{(-1)^{k}}{P_{k} P_{k+1}}\right)^{-1}\right\rfloor=-P_{2 n}-1
$$
3.7.

According to (3.10) and (3.13), we established the theorem

IV. MAIN Results III: The RECIPROCAL SuMs OF EVEN AND ODD TERMS

$$
\begin{aligned}
& \text { Theorem } 4.1 \text { If } m \geq 3 \text { and } n \geq 1, \\
& \left\lfloor\left(\sum_{k=n}^{m n} \frac{1}{P_{2 k}}\right)^{-1}\right\rfloor=2 P_{2 n-1}-1
\end{aligned}
$$

Proof applying (2.1), or $k \geq 2$

$\frac{1}{2 P_{2 k-1}-1}-\frac{1}{P_{2 k}}-\frac{1}{2 P_{2 k+1}-1}=\frac{2\left(P_{2 k+1}-P_{2 k-1}\right) P_{2 k}-\left(2 P_{2 k-1}-1\right)\left(2 P_{2 k+1}-1\right)}{\left(2 P_{2 k-1}-1\right) P_{2 k}\left(2 P_{2 k+1}-1\right)}=\frac{2 P_{2 k-1}+2 P_{2 k+1}-5}{\left(2 P_{2 k-1}-1\right) P_{2 k}\left(2 P_{2 k+1}-1\right)}>0$

$$
\frac{1}{2 P_{2 k-1}-1}-\frac{1}{P_{2 k}}-\frac{1}{2 P_{2 k+1}-1}=\frac{2 P_{2 k-1}+2 P_{2 k+1}-5}{\left(2 P_{2 k-1}-1\right) P_{2 k}\left(2 P_{2 k+1}-1\right)}>0
$$

Which implies that

$\frac{1}{P_{2 k}}=\frac{1}{2 P_{2 k-1}-1}-\frac{1}{2 P_{2 k+1}-1}-\frac{2 P_{2 k-1}+2 P_{2 k+1}-5}{\left(2 P_{2 k-1}-1\right) P_{2 k}\left(2 P_{2 k+1}-1\right)}<\frac{1}{2 P_{2 k-1}-1}-\frac{1}{2 P_{2 k+1}-1}$,

So

$$
\sum_{k=n}^{m n} \frac{1}{P_{2 k}}<\frac{1}{2 P_{2 n-1}-1}-\frac{1}{2 P_{2 m n+1}-1}<\frac{1}{2 P_{2 n-1}-1}
$$

From (2.1) we obtain

$\frac{1}{2 P_{2 k-1}}-\frac{1}{P_{2 k}}-\frac{1}{2 P_{2 k+1}}=\frac{P_{2 k+1} P_{2 k}-2 P_{2 k-1} P_{2 k+1}-P_{2 k-1} P_{2 k}}{2 P_{2 k-1} P_{2 k} P_{2 k+1}}=\frac{-1}{P_{2 k-1} P_{2 k} P_{2 k+1}}$

Hence

$$
\frac{1}{P_{2 k}}=\frac{1}{2 P_{2 k-1}}-\frac{1}{2 P_{2 k+1}}+\frac{1}{P_{2 k-1} P_{2 k} P_{2 k+1}}
$$

Therefore

$$
\sum_{k=n}^{m n} \frac{1}{P_{2 k}}=\frac{1}{2 P_{2 n-1}}-\frac{1}{2 P_{2 m n+1}}+\sum_{k=n}^{m n} \frac{1}{P_{2 k-1} P_{2 k} P_{2 k+1}}>\frac{1}{2 P_{2 n-1}}-\frac{1}{2 P_{2 m n+1}}+\frac{1}{P_{2 n-1} P_{2 n} P_{2 n+1}}
$$

For all $n \geq 1, m \geq 3$, with (2.2)

$$
P_{m-1} P_{n}+P_{m} P_{n+1}=P_{m+n}, \quad P_{m+n}>P_{m} P_{n+1}>P_{m} P_{n}
$$

Which implies that

$$
P_{2 n-1} P_{2 n} P_{2 n+1}<P_{6 n+1}<P_{2 m n+1},
$$

With (4.4)

$$
\sum_{k=n}^{m n} \frac{1}{P_{2 k}}>\frac{1}{2 P_{2 n-1}}-\frac{1}{2 P_{2 m n+1}}+\frac{1}{P_{2 n-1} P_{2 n} P_{2 n+1}}>\frac{1}{2 P_{2 n-1}}
$$
4.1.

According to (4.2) and (4.5), we established the theorem Corollary.4.2

$$
\left\lfloor\left(\sum_{k=n}^{\infty} \frac{1}{P_{2 k}}\right)^{-1}\right\rfloor=2 P_{2 n-1}-1 .
$$

Theorem 4.3 If $m \geq 2$ and $n \geq 1$,

$$
\left\lfloor\left(\sum_{k=n}^{m n} \frac{1}{P_{2 k-1}}\right)^{-1}\right\rfloor=2 P_{2 n-2} .
$$

Proof applying (2.1), or $k \geq 2$

$\frac{1}{2 P_{2 k-2}}-\frac{1}{P_{2 k-1}}-\frac{1}{2 P_{2 k}}=\frac{P_{2 k-1} P_{2 k}-2 P_{2 k-2} P_{2 k}-P_{2 k-2} P_{2 k-1}}{2 P_{2 k-2} P_{2 k-1} P_{2 k}}=\frac{1}{P_{2 k-1} P_{2 k} P_{2 k+1}}>0$

$$
\frac{1}{P_{2 k-1}}=\frac{1}{2 P_{2 k-2}}-\frac{1}{2 P_{2 k}}-\frac{1}{P_{2 k-2} P_{2 k-1} P_{2 k}}<\frac{1}{2 P_{2 k-2}}-\frac{1}{2 P_{2 k}}
$$

$$
\sum_{k=n}^{m n} \frac{1}{P_{2 k-1}}<\frac{1}{2 P_{2 n-2}}-\frac{1}{2 P_{2 m n}}<\frac{1}{2 P_{2 n-2}}
$$

$\frac{1}{2 P_{2 k-2}+1}-\frac{1}{P_{2 k-1}}-\frac{1}{2 P_{2 k}+1}=\frac{2\left(P_{2 k}-P_{2 k-2}\right) P_{2 k-1}-\left(2 P_{2 k-2}+1\right)\left(2 P_{2 k}+1\right)}{\left(2 P_{2 k-2}+1\right) P_{2 k-1}\left(2 P_{2 k}+1\right)}=\frac{2^{2}-2 P_{2 k-2}-2 P_{2 k}-1}{\left(2 P_{2 k-2}+1\right) P_{2 k-1}\left(2 P_{2 k}+1\right)}<0$

$$
\sum_{k=n}^{m n} \frac{1}{P_{2 k-1}}=\frac{1}{2 P_{2 n-2}+1}-\frac{1}{2 P_{2 m n}+1}+\sum_{k=n}^{m n} \frac{2 P_{2 k-2}+2 P_{2 k}+1-2^{2}}{\left(2 P_{2 k-2}+1\right) P_{2 k-1}\left(2 P_{2 k}+1\right)},
$$

$$
\sum_{k=n}^{m n} \frac{1}{P_{2 k-1}}>\frac{1}{2 P_{2 n-2}+1}-\frac{1}{2 P_{2 m n}+1}+\frac{2 P_{2 n-2}+2 P_{2 n}+1-2^{2}}{\left(2 P_{2 n-2}+1\right) P_{2 n-1}\left(2 P_{2 n}+1\right)},
$$


By reason of

$\frac{2 P_{2 n-2}+2 P_{2 n}+1-2^{2}}{\left(2 P_{2 n-2}+1\right) P_{2 n-1}\left(2 P_{2 n}+1\right)}>\frac{2 P_{2 n}+1}{\left(2 P_{2 n-2}+1\right) P_{2 n-1}\left(2 P_{2 n}+1\right)}>\frac{1}{\left(2 P_{2 n-2}+1\right) P_{2 n-1}}>\frac{1}{2 P_{4 n}+1}>\frac{1}{2 P_{2 n n}+1}$,

Hence

$$
\sum_{k=n}^{m n} \frac{1}{P_{2 k-1}}>\frac{1}{2 P_{2 n-2}+1}
$$

According to (4.7) and (4.8), the theorem 4.3 is true.

$$
\text { Corollary } 4.4\left\lfloor\left(\sum_{k=n}^{\infty} \frac{1}{P_{2 k-1}}\right)^{-1}\right\rfloor=2 P_{2 n-2}
$$

Theorem 4.5 If $m \geq 2$ and $n \geq 1$,

$$
\left\lfloor\left(\sum_{k=n}^{m n} \frac{1}{P_{2 k}^{2}}\right)^{-1}\right\rfloor=2 P_{4 n-2}-1 .
$$

Proof applying (2.1) and (2.4)

$$
\begin{gathered}
P_{2 k}^{2}-P_{2 k-2}^{2}=2 P_{4 k-2}, \\
P_{2 k+2}^{2}-P_{2 k}^{2}=2 P_{4 k+2}, \\
P_{2 k-1}^{2} P_{2 k+1}^{2}-P_{2 k-2}^{2} P_{2 k+2}^{2}=5\left(P_{2 k}^{2}+P_{2 k-1} P_{2 k+1}-4\right),
\end{gathered}
$$

According to (4.9), (4.10) and (4.11)

$\frac{1}{2 P_{2 k-2}}-\frac{1}{P_{2 k-1}}-\frac{1}{2 P_{2 k}}=\frac{P_{2 k-1} P_{2 k}-2 P_{2 k-2} P_{2 k}-P_{2 k-2} P_{2 k-1}}{2 P_{2 k-2} P_{2 k-1} P_{2 k}}=\frac{1}{P_{2 k-1} P_{2 k} P_{2 k+1}}>0$

Hence

$$
\frac{1}{2 P_{4 k-2}-1}-\frac{1}{P_{2 k}^{2}}-\frac{1}{2 P_{4 k+2}-1}>0
$$

Therefore

$\sum_{k=n}^{m n} \frac{1}{P_{2 k}^{2}}<\frac{1}{2 P_{4 n-2}-1}-\frac{1}{2 P_{4 m n+2}-1}<\frac{1}{2 P_{4 n-2}-1}$

For all $k \geq 2$,

$$
\begin{aligned}
\frac{1}{2 P_{4 k-2}}-\frac{1}{P_{2 k}^{2}}-\frac{1}{2 P_{4 k+2}} & =\frac{P_{2 k}^{2}\left(P_{4 k+2}-P_{4 k-2}\right)-2 P_{4 k-2} P_{4 k+2}}{2 G_{4 k-2} G_{2 k}^{2} G_{4 k+2}} \\
& =\frac{-\left(4 P_{2 k}^{2}-4 P_{2 k-1} P_{2 k+1}\right)}{P_{4 k-2} P_{2 k}^{2} P_{4 k+2}}<\frac{-1}{P_{4 k-2} P_{4 k+2}}<0
\end{aligned}
$$

From which we obtain

$\sum_{k=n}^{m n} \frac{1}{P_{2 k}^{2}}>\frac{1}{2 P_{4 n-2}}-\frac{1}{2 P_{4 m n+2}}+\sum_{k=n}^{m n} \frac{1}{P_{4 k-2} P_{4 k+2}}>\frac{1}{2 P_{4 n-2}}-\frac{1}{2 P_{4 m n+2}}+\frac{1}{P_{4 n-2} P_{4 n+2}}$

Applying (2.4)

$2 P_{8 n+2}=P_{4 n+2}^{2}-P_{4 n}^{2}=2 P_{4 n+1}\left(P_{4 n+2}+P_{4 n}\right)>2 P_{4 n+1} P_{4 n+2}>2 P_{4 n+2} P_{4 n-2}$

So

$$
\sum_{k=n}^{m n} \frac{1}{P_{2 k}^{2}}>\frac{1}{2 P_{4 n-2}}
$$

Combining (4.13) and (4.15), we have theorem 4.5

$$
\text { Corollary } 4.6\left\lfloor\left(\sum_{k=n}^{\infty} \frac{1}{P_{2 k}^{2}}\right)^{-1}\right\rfloor=2 P_{4 n-2}-1 \text {. }
$$

Theorem 4.7 If $m \geq 2$ and $n \geq 1$, $\left\lfloor\left(\sum_{k=n}^{m n} \frac{1}{P_{2 k-1}^{2}}\right)^{-1}\right\rfloor=2 P_{4 n-4}$.

Proof by the calculation of (2.4), we obtain, $k \geq 2$

$$
\begin{gathered}
P_{2 k+1}^{2}-P_{2 k-1}^{2}=2 P_{4 k} \\
P_{2 k-1}^{2}-P_{2 k-3}^{2}=2 P_{4 k-4}
\end{gathered}
$$

From (4.16) and (4.17)

$$
\begin{aligned}
& P_{2 k-1}^{2}\left(P_{4 k}-P_{4 k-4}\right)-2 P_{4 k} P_{4 k-4} \\
& =\frac{1}{2} P_{2 k-1}^{2}\left(P_{2 k+1}^{2}-2 P_{2 k-1}^{2}+P_{2 k-3}^{2}\right)-\frac{1}{2}\left(P_{2 k+1}^{2}-P_{2 k-1}^{2}\right)\left(P_{2 k-1}^{2}-P_{2 k-3}^{2}\right) \\
& =\frac{1}{2}\left(P_{2 k+1} P_{2 k-3}-P_{2 k-1}^{2}\right)\left(P_{2 k+1} P_{2 k-3}+P_{2 k-1}^{2}\right) \\
& =2\left(P_{2 k+1} P_{2 k-3}+P_{2 k-1}^{2}\right)
\end{aligned}
$$

By (4.16), (4.17) and (4.18)

$$
\frac{1}{2 P_{4 k-4}}-\frac{1}{P_{2 k-1}^{2}}-\frac{1}{2 P_{4 k}}=\frac{P_{2 k-1}^{2}\left(P_{4 k}-P_{4 k-4}\right)-2 P_{4 k} P_{4 k-4}}{2 P_{4 k-4} P_{2 k-1}^{2} P_{4 k}}=\frac{P_{2 k+1} P_{2 k-3}+P_{2 k-1}^{2}}{P_{4 k-4} P_{2 k-1}^{2} P_{4 k}}>0
$$


Which implies

$$
\frac{1}{P_{2 k-1}^{2}}<\frac{1}{2 P_{4 k-4}}-\frac{1}{2 P_{4 k}}
$$

Hence

$$
\begin{gathered}
\sum_{k=n}^{m n} \frac{1}{P_{2 k-1}^{2}}<\frac{1}{2 P_{4 n-4}}-\frac{1}{2 P_{4 m n}}<\frac{1}{2 P_{4 n-4}} \\
\frac{1}{2 P_{4 k-4}+1}-\frac{1}{P_{2 k-1}^{2}}-\frac{1}{2 P_{4 k}+1} \\
=\frac{2\left(P_{4 k}-P_{4 k-4}\right) P_{2 k-1}^{2}-\left(2 P_{4 k-4}+1\right) 2\left(a P_{4 k}+1\right)}{\left(2 P_{4 k-4}+1\right) P_{2 k-1}^{2} 2\left(a P_{4 k}+1\right)} \\
=\frac{2^{2}\left(P_{2 k+1} P_{2 k-3}+P_{2 k-1}^{2}\right)-\left(P_{2 k+1}^{2}-P_{2 k-3}^{2}\right)-1}{\left(2 P_{4 k-4}+1\right) P_{2 k-1}^{2}\left(2 P_{4 k}+1\right)}
\end{gathered}
$$

By reason of

$$
\begin{gathered}
P_{2 k+1} P_{2 k-3}+P_{2 k-1}^{2}=\left(2 P_{2 k}-P_{2 k-1}\right)\left(P_{2 k-1}-2 P_{2 k-2}\right)+P_{2 k-1}^{2}=5 P_{2 k-1}^{2}+4, \\
P_{2 k+1}^{2}-P_{2 k-3}^{2}=12 P_{2 k-1}\left(P_{2 k}+P_{2 k-2}\right)>24 P_{2 k-1}^{2}+16,
\end{gathered}
$$

Therefore

$$
2^{2}\left(P_{2 k+1} P_{2 k-3}+P_{2 k-1}^{2}\right)-\left(P_{2 k+1}^{2}-P_{2 k-3}^{2}\right)-1<-P_{2 k-1}^{2} .
$$

So

$$
\frac{1}{2 P_{4 k-4}+1}-\frac{1}{P_{2 k-1}^{2}}-\frac{1}{2 P_{4 k}+1}<\frac{-1}{\left(2 P_{4 k-4}+1\right)\left(2 P_{4 k}+1\right)}<0
$$

$$
\frac{1}{P_{2 k-1}^{2}}>\frac{1}{2 P_{4 k-4}+1}-\frac{1}{2 P_{4 k}+1}+\frac{1}{\left(2 P_{4 k-4}+1\right)\left(2 P_{4 k}+1\right)}
$$

$$
\begin{aligned}
\sum_{k=n}^{m n} \frac{1}{P_{2 k-1}^{2}} & >\frac{1}{2 P_{4 n-4}+1}-\frac{1}{2 P_{4 m n}+1}+\sum_{k=n}^{m n} \frac{1}{\left(2 P_{4 k-4}+1\right)\left(2 P_{4 k}+1\right)} \\
& >\frac{1}{2 P_{4 n-4}+1}-\frac{1}{2 P_{4 m n}+1}+\frac{1}{\left(2 P_{4 n-4}+1\right)\left(2 P_{4 n}+1\right)}
\end{aligned}
$$

Also because

$$
\left(2 P_{4 n-4}+1\right)\left(2 P_{4 n}+1\right)=2^{2} P_{4 n-4} P_{4 n}+2 P_{4 n-4}+2 P_{4 n}+1<P_{8 n}+1 .
$$

$$
\sum_{k=n}^{m n} \frac{1}{P_{2 k-1}^{2}}>\frac{1}{2 P_{4 n-4}+1}
$$

Combining (4.19) and (4.21), the statement is true

$$
\left\lfloor\left(\sum_{k=n}^{m n} \frac{1}{P_{2 k-1}^{2}}\right)^{-1}\right\rfloor=2 P_{4 n-4}
$$

Corollary $4.8\left\lfloor\left(\sum_{k=n}^{\infty} \frac{1}{P_{2 k-1}^{2}}\right)^{-1}\right\rfloor=2 P_{4 n-4}$.

\section{ACKNOWLEDGEMENT}

This research was financially supported by the Special projects of scientific research of education department of Shaanxi Provincial (15JK1262).

\section{REFERENCES}

[1] Ohtsuka H, Nakamura S. On the sum of reciprocal Fibonacci numbers[J]. Fibonacci Quart.46/47(2008/2009),153-159.

[2] Wang AYZ, Wen PB. On the partial finite sums of the reciprocals of the Fibonacci numbers[J] . Inequal.Appl.2015, (1),1-13. DOI : 10.1186/s13660-015-0595-6.

[3] Wang AYZ, Zhang F. The reciprocal sums of even and odd terms in the Fibonacci sequence[J]. Inequal.Appl.2015 (1),1-13.DOI :10.1186/s13660-015-0902-2.

[4] Wang AYZ, Liu RN. Sums of products of two reciprocal Fibonacci numbers[J]. Adv.Differ.Equ.2016, 2016(1),1-26. DOI: 10.1186/s13662-016-0860-0.

[5] Wang,AYZ,Zhang,F.The reciprocal sums of the Fibonacci3-subsequences[J].Adv.Differ.Equ.2016-2016(1),1-15. DOI:10.1186/s13662-016-0761-2.

[6] SarahH. Holliday, Takao. Komatsu. On the Sum of Reciprocal Generalized Fibonacci Numbers[J] .Integers.2011,11(4),441-455.

[7] Z. Wenpeng and W.Tingting.The infinite sum of reciprocal Pell numbers $[\mathrm{J}]$. Applied Mathematics and Computation, vol. 218, no. 10, pp. 6164-6167, 2012.

[8] W. Zhang and T. Wang. The infinite sum of reciprocal of the square of the Pell numbers[J]. Journal of Weinan Teacher's University, vol. 26, pp. 39-42, 2011.

[9] Z. Xu and T. Wang. The infinite sum of the cubes of reciprocal Pell numbers, [J]. Advances in Difference Equations, vol. 2013, article 184, 2013. 